\title{
No-alineación, un vistazo histórico y conceptual
}

\begin{abstract}
Boja:A TADIc es Research Fellow en el Departamento de Relaciones Internacionales del Instituto de Politica y Economia Internacional, de la Universidad de Belgrado. Se graduó en Filosofia y luego obtuvo el doctorado en Ciencia Politica en esa misma universidad. Entre sus publicaciones se cuentan The German Question in the Postwar Period, monografia publicada por el Instituto de Politica y Economia Internacional (Belgrado, 1960), The Subjet of the Study of International Relations, un capitulo del libro The Sludy of Inlemational Relalions Theory' in the West, publicado por el mismo Instituto (Belgrado, 1965), Nonaligned Countries and the Postwar Negotiations on Disarmament, un capitulo del libro Disarmament (Belgrado, j967). Prepara la edición de Nonalignement, a Historical Survey (1946-1966). El presente trabajo fue presentado a la Conferencia sobre No-alincación yealizada en Novisad, Yugoslavia, en enero de 1969 . Se publica con la autorización de su autora.
\end{abstract}

Son numerosas las consideraciones semánticas cuya meta es delimitar y demarcar con precisión el significado de los diferentes términos que se emplean en la literatura de ciencia politica para denotar el concepto de no-alineación. Estas consideraciones muy a menudo terminan por hacer notar que no se ha encontrado aún el término adecuado, porque ninguno de los existentes logra definir verdaderamente el fenómeno. Se puede decir que esto no es consecuencia de una falta de habilidad para encontrar una palabra, sino, y sobre todo, consecuencia de que la misma noalineación es todavia una categoria insuficientemente clara dentro de la teoria de las relaciones internacionales. Se han hecho muchos intentos para delimitar con mayor agudeza el concepto de la no-alineación, pero hasta la fecha nadie ha logrado producir una definición por todos aceptada. No sólo hay perspectivas diferentes respecto a este fenómeno, sino también diversos modos de aproximarse a él, que resultan del énfasis que 
Bojana Tadić / No-alinención, un vistazo histórico y conceptual se pone en algunas de sus caracteristicas, en los diferentes elementos que lo constituyen, y de una diferente evaluación ideológica.

Dentro de los diversos intentos que se han hecho para responder las preguntas sobre lo que la no-alineación es, sobre qué tipo de fenómeno de las relaciones internacionales se trata $y$ sobre cuáles sean sus componentes básicos, por medio de un proceso de eliminación es posible distinguir tres formas de pensar, que no siempre son mutuamente excluyentes, aunque a menudo dan nacimiento a interpretaciones diferentes.

Según la primera de estas interpretaciones, sustentada en sus diversas variantes por autores tales como C. Crabb ${ }^{1}, \mathrm{~K} . \mathrm{Babaa}^{2}, \mathrm{M}$. Legum ${ }^{3}$ $y$ otros, la no-alineación es una doctrina de politica exterior, una Weltanschauung ideológica, respecto a las relaciones internacionales, un sistema de nociones sobre este mundo. Aunque refleje la realidad de los acontecimientos de las relaciones internacionales, según este concepto, la no-alineación es sobre todo una construcción racionante, con marcadas caracteristicas filosóficas e ideológicas. Pertenece a la esfera de los desiderata $y$ de las visiones sobre el mundo en que existimos. Como tal, la no-alineación no es considerada como una politica exterior, con los atributos propios de una politica - metas claramente definidas, acción organizada para implementar esas metas y un cierto grado de enlenle entre los que sustentan dicha politica. Algunos de los que apoyan este punto de vista miran a la filosofia de la no-alineación como una suerte de fuerza en la debilidad $^{4}$, y' por eso mismo un sistema de concepciones no suficientemente coherente, no del todo lógico ni exacto, una suma de conceptos que a menudo se defienden a si mismos con argumentos insuficientemente claros. Algunos de estos autores miran a la no-alineación no tanto como algo original, sino más bien como una doctrina eminentemente ecléctica y pragmática. Sin embargo, a pesar de estas caracteristicas, en la literatura se esboza que la filosofia de la no-alineación expresa las reales

${ }^{1}$ C. Crabb, Nonalignment in Ilorld Iffairs, Introduction, The Annals of the American Academy of Political and Social Science, November ig6j, p. 4 .

${ }^{2} \mathrm{~K}$. Babaa, C. Crabb, Nonalignment as a Diplomalic and Ideological Creto, The Annals of the American Academy of Political and Social Science, November 1965, pp. 6-17.

3.Margaret Legum, Africn and . Vonalignment, . Vonalignment, editado por .J. II. Burton, Londres, Andre Deutsch, ig66, pp. 56-57.

${ }^{4}$ C. Crabb. The Elcphants and the Grass - A Study of Nonalignment, New lork. Frederick .1. Praeger, 1965, pp. $82-8.1$. 
E S T L D I O S I $\therefore$ T ER $\therefore A C I O N A L E S$

necesidades de un gran número de paises en el mundo contemporáneo, lo que la hace atractiva y fácilmente aceptable ${ }^{5}$.

Otra respuesta a la pregunta por lo que la no-alineación es, y que se encuentra con muchisima frecuencia en la literatura existente, sugiere que es un determinado tipo de politica exterior, o más bien una suma de idénticas o similares orientaciones para la politica exterior de un determinado número de paises en el mundo.

También este tipo de respuesta tiene un amplio número de variantes. En la literarura teórica, encontramos a la no-alineación expresada en términos de una politica a corto plazo, cuya meta seria el aprovechamiento de las ventajas que surgen de la negativa a unirse con los bloques de poder; una politica cuyos lideres están insuficientemente entrenados en las técnicas de coordinación entre las politicas exteriores y los intereses nacionales, en otras palabras, una carencia de tradición y una falta de conocimiento, que se habria hecho patente en los gestores de la politica de los paises más recientemente liberados; la no-alineación es sólo el rechazo de un pais de juntarse a los grupos militares del Este o del Oeste, y el rechazo de aceptar como propio el conflicto entre los bloques de poder. Uno se cruza también con la noción de la no-alineación como sinónimo de un tipo de politica exterior con un status de independencia en las relaciones internacionales, una politica con metas claramente definidas, hechas sobre todo para preservar la paz mundial -un prerrequisito para la afirmación de la independencia nacional de los paises recientemente liberados y de su acelerado desarrollo económico.

Por último, requiere una especial atención un tercer tipo de respuesta, que ha sido elaborada en la literatura teórica por el profesor Burton. No se opone a las perspectivas anteriormente mencionadas que miran la no-alineación como una filosofia politica o como una politica exterior, pero aporta un sentido cualitativamente diferente y más amplio.

Después de establecer que en el presente, en la era nuclear del desarrollo de la civilización mundial, ha habido cambios esenciales en las relaciones internacionales, el profesor Burton propone la teoria de la noalineación como un sistema nuevo de relaciones, que corresponde a esos cambios, una solución a largo plazo y la alternativa al sistema basado en el poder, el cual está llegando a ser históricamente cada dia más obsoleto. De acuerdo a esta interpretación, la no-alineación no es sólo una

sp. Lyion, . Neutralism, Leicester L'niversity Press, 1963, pp. 59-88. 
Bojna Tardic / No-alineación, un vistazo histórico y conceptual

politica exterior de uno o varios paises del mundo. Constituye un nuevo valor, un nuevo sistema internacional que se ha creado, no como un objetivo especifico de las politicas nacionales de los paises no comprometidos, sino como un resultado inesperado de la politica que cada pais noalineado ha realizado separada o conjuntamente con otro. La no-alineación, en consecuencia, crea un nuevo esquema de relaciones internacionales, las que, en un análisis final, trascienden cualquier objetivo individual de un pais no-alineado, y constituyen un modelo alternativo para el sistema tradicional de poder. Aunque, de acuerdo con esta opinión, da una respuesta a los intereses concretos de los paises que la crearon, la noalineación contiene ciertos elementos de estabilidad que la hacen un fenómeno permanente en las relaciones internacionales. El sistema que la no-alineación ha creado está basado en los sistemas de cooperación, lazos mutuos y competencia, como forma de relación entre los paises vinculados, en las condiciones de la tecnologia moderna de la segunda mitad del siglo $\mathrm{xx}^{6}$.

Sin entrar en un análisiśs más profundo de los mencionados intentus para definir este fenómeno, trataremos de señalar una de las posibles direcciones para investigar su contenido. Parece necesario definir aqui las condiciones en que este fenómeno se produjo, que encontraron su correspondiente lugar en sus caracteristicas básicas.

Considerada en su perspectiva histórica, la no-alineación es un fenómeno reciente en las relaciones internacionales. Surgió inmediatamente después de la Segunda Guerra Mundial, como resultado de la existencia y fusión de una multitud de procesos simultáneos de los cuales dos tuvieron una abrumadora significación.

Una caracteristica esencial de las relaciones internacionales de postguerra, que tuvo una influencia directa sobre la no-alineación, fue el proceso de polarización de los estados miembros de la coalición antihutleriana, en la forma de sistemas estatales politico-militares opuestos entre si respecto a problemas ideológicos, politicos y militares, con fuertes tendencias a expandir sus esferas de influencia y a dividir el mundo en dos bloques de poder. Las tendencias dirigidas a asimilar o a unir los estados, fuera de Europa o Norteamérica, al grupo de estados orientales

6. W. Burton, International Relations - A General Theory, Cambridge Liniversity Press, 1965 , pp. 16. -168, 231-232. 
$y$ occidentales, fueron sólo factores de un proceso de bipolarización, en otras palabras, antecedentes para la realización de su total impacto.

Simultáneo al proceso de formación de bloques, se produjo también el proceso de la desintegración del sistema colonial, acompañado por la aparición de una multitud de nuevos estados independientes, primero en el continente asiático y luego en Africa. Este proceso en si mismo significó la negación de la tendencia a dividir el mundo en dos bloques. Los estados soberanos recién creados, por el hecho de su real aparición, estaban destinados a oponerse a esta tendencia y a introducir en la comunidad internacional una nueva perspectiva sobre el mundo, cualitativamente diferente de los conceptos existentes de grandes potencias, una nueva perspectiva basada en los intereses nacionales de los nuevos paises que tenian elementos similares suficientes, pero que al mismo tiempo no coincidian con los intereses de los bloques.

La no-alineación apareció y se desarrolló en el marco de la existencia de estas tendencias contradictorias, y es por consiguiente en si misma un fenómeno muy complejo. Su elemento básico es una posición independiente en los asuntos internacionales $y$, en consecuencia, una actitud negativa hacia la alineación con los bloques y hacia las consecuencias que las politicas de bloque acarrean.

La no-alineación se produjo como una reacción a las politicas de poder y a la división existente en el mundo. Con este componente - la no-alineación- desde el principio contrapesó las tendencias hacia una división final del mundo que disminuiria la individualidad politica de los paises que estaban vinculados o incluidos en los sistemas de bloque de los estados, y subordinados a los intereses de la comunidad, o más bien a aquellos del gran poder dirigente.

Otro componente esencial de la no-alineación, inseparable del primero y que representa su contenido positivo, es la lucha por la realización de los principios de coexistencia activa. Estos principios, formulados en el texto de la Declaración de las Naciones Unidas, constituyen la base de los esfuerzos desarrollados por los paises no-alineados para disminuir las contradicciones en las esferas económicas, politicas y sociales de las relaciones internacionales, y permitir la creación de un nuevo orden internacional basado en la igual cooperación entre naciones y estados, prescindiendo de las diferencias geográficas o demográficas, de la organización politica o de los tipos de civilización. 
Bojana Taflić / Nonalincación, un vistazo histórico y conceptual

Estos dos factores tomados en conjunto, oposición a divisiones y excesos de las politicas de bloque por una parte, y lucha por un nuevo valor en las relaciones entre estados - como estan establecidos en los principios de coexistencia pacifica activa - por otra, pueden ser considerados componentes esenciales de la no-alineación

Definir lo esencial de este fenómeno como una combinación de los dos elementos mencionados no significa, sin embargo, que a través de la historia de la no-alineación ellos aparecieron en idéntica forma y con la misma intensidad. Aun cuando estos elementos están permanentemente presentes en ciertos periodos y especialmente en las condiciones de intensa confrontación de bloques, hubo un pronunciado énfasis en la primera caracteristica antibloque de la no-alineación, y ella dominó la politica exterior de los paises no-alineados. Sucedió esto generalmente en los periodos de crisis, en las épocas de deterioro en las relaciones de las grandes potencias, o en etapas de pronunciada presión sobre los paises recientemente liberados no-alineados. En estas condiciones el deseo de autodefensa, para neutralizar las presiones externas $y$ los esfuerzos por disminuir la amenaza de un conflicto directo entre las potencias dominantes de bloque, que podian exponer aún la independencia de los estados fuera de estas agrupaciones, exigieron una politica definitiva antibloque, lo que en tales condiciones llegó a ser un componente dominante de la no-alineación.

Contrario a esto, en los periodos de reducida tensión entre las potencias lidere:s de bloque, lo que sucedió cada cierto tiempo en el desarrollo de postguerra de las relaciones internacionales, y en la politica exterior de los paises que estaban menos expuestos a la presión directa de las grandes potencias, hubo tendencia a una insistencia mayor sobre la necesidad de poner en práctica los principios de la coexistencia pacifica $y$ de la cooperación activa en las relaciones entre estados, y a cambiar las existentes formas de comunicación entre los estados, los que en la mayoria de los casos se basaban en politicas de poder y en formas varias de coerción $y^{\prime}$ presión.

Esta tendencia es obvia si se hace un análisis comparativo de las doctrinas y de la actividad de politica exterior de los paises no-alineados en ciertos periodos caracteristicos en el desarrollo de las relaciones internacionales desde la guerra. A principios de la década del cincuenta, en la época en que la diferenciación de bloques alcanzó un grado muy alto, la 
posición no comprometida se destacó en forma prominente en los documentos oficiales de politica exterior de los paises no-alineados con las potencias de bloque. A mediados de la década del cincuenta, en la épocia de la Conferencia de Bandung de los estados afroasiáticos, la gradual estabilización en las relaciones entre bloques coincidió con la insistencia de los paises asiáticos y africanos sobre la necesidad de la accptación universal de los principios de coexistencia pacifica, como se definió en el documento final de esta reunión. Puede llegarse a una conclusión similar si se hace una comparación entre los documentos de la Conferencia de Belgrado de los paises no-alineados, efectuada durante un periodo critico en las relaciones de los grandes poderes, y los de la Conferencia del Cairo, la cual a su propia manera reflejó el relajamiento de las tensiones en las relaciones internacionales y el cambio de los conflictos directos de bloques en choques indirectos en diferentes puntos del hemisferio, tales como Laos, Vietnam del Sur, el Congo y otros.

Este cambio de énfasis sobre uno u otro componente de la no-alineación dependiente del grado de la tensión internacional es, sin embargo, más digno de atención como una tendencia que como algo absolutamente inevitable, pues los ejemplos ya mencionados u otros ejemplos no serian suficientes para probar sin posibilidad de duda eslabones causales entre estos fenómenos, aunque hay ciertamente una coincidencia, que pliede no ser accidental. Además, estuvo presente, en menor medida, en el periodo de desarrollo más reciente de la no-alineación, en el que la situación es más compleja y el fenómeno ha ido desapareciendo.

Sin ir más allá de esta tesis nos detendremos sobre nuestro descubrimiento inicial acerca de la no-alineación como una sintesis de los elementos mencionados. En si mismos son muy complejos y están ligados en forma tan dependientes y reciproca unos de otros, que es frecuente en la práctica encontrar una gran dificultad para separarlos.

Cercano a tal interrogante, está también el problema de definir los paises no-alineados como sujetos del fenómeno de la no-alineación. Esto es también muy complejo $y^{\prime}$ dificil de resolver. Lo que es obvio, sin embargo, es el hecho que los paises no-alineados son una categoria politica de paises que en la mayoria de los casos han sido recientemente liberados, $y$ que apoyan más o menos activamente los programas adoptados en las conferencias de paises no comprometidos, o que explicitamente se declaran en favor de la no-alineación en las relaciones internacionales. 
Bojana Tadić / No-alincación, un vistazo histórico y conceptual

Esta categoria de paises tiene un componente económico común clarilmente enunciado, en tanto cuanto la gran mayoria de ellos pertenece al grupo de los paises en desarrollo, aunque estos dos conceptos no son idénticos. No todos los paises no-a lineados son paises en vias de desarrollo; pero si lo son los más activos entre ellos, lo que en la mayoria de los casos en forma colectiva o individual inician a aunan acciones politicas para implementar las politicas adoptadas:

Los paises no-alineados nunca han representado, sin embargo, un grupo formal e institucionalizado de estados y por consiguiente claramente definido. Su número ha variado con pronunciada tendencia al crecimiento, porque la mayoria de los antiguos paises coloniales, una vez que adquirieron independencia, optaron por esta posición en su orientación en politica exterior, obteniendo cierto éxito al ponerla en práctica. Por otra parte si prescindimos de la asistencia a las conferencias de los paises no-alinendos, por considerarlo un criterio insuficientemente confiable para determinar el status de un pais no-alineado, seria dificil delinear un limite seguro entre los paises no-alineados $y$ aquellos que estando en vias de desarrollo no entran en dicha categoria, exceptuándose los que no forman parte directa de los bloques politico-militares. Además, en estos últimos como en algunos otros, las tendencias hacia una más completa independencia y no-alineación, no sólo aparecen sino que claramente se han ido fortaleciendo, especialmente en los años recientes, pero las posibilidades en algunos de ellos, debido a todo tipo de circunstancias de naturaleza interna y externa, son tadavia relativamente restringidas. Como resultado de todo esto, la no-alineación desde el punto de vista de sus sujetos es una categoria muy amplia, insuficientemente exacta y en constante cambio.

Habiendo asi examinado la no-alineación descle el punto de vista de su naturaleza $y$ de las caracteristicas de aquellos que la practican, procederemos a bosquejar el desarrollo de este fenómeno hasta hoy dia. Enfatizaremos los rasgos generales que la no-alineación muestra en diferentes periodos, más que las numerosas formas especificas en que apareció, apoyándonos en las diferentes condiciones e intereses nacionales concretos de cada pais por separado.

En vista del alcance de la no-alineación, a saber, el número de paises que han adoptado esta idea, sus rasgos básicos y la intensidad de su acción, el desa- 
rrollo histórico de este fenómeno, con reservas en contra de los peligros de la esquematización $y$ simplificación inherentes a la periodicidad de los eventos sociales, podria ser dividido en varios periodos.

El primer periodo, que fue también el periodo de su formación, cubre el tiempo comprendido entre el término de la Segunda Guerra Mundial y la Conferencia de Bandung de los Paises Afroasiáticos, en I955. Se caracterizó por el número relativamente pequeño de paises que la asumieron $y$ por el propósito de permanecer alejados, a cualquier precio, de las potencias de bloque y su politica de quedar fuera del área de los intereses de bloque; de rechazar identificarse con sus conflictos, $y$ en cierta medida, de adoptar una actitud separatista concentrándose en sus propios problemas o en aquellos de sus regiones o de paises vecinos. En este periodo los paises no-alineados no estaban suficientemente unidos, de modo que su influencia en las relaciones internacionales fue rara vez sentida. La orientación de la no-alineación en las politicas exteriores dio frecuentes muestras de politicas exteriores nacionales individuales, $y$ fueron escasas las acciones conjuntas de los paises no-alineados. Las grandes potencias en este periodo prestaron relativamente poca atención al fenómeno, creyendo que los-paises recién liberados no tenian más alternativa que tomar un partido en la Guerra Fria, e hicieron esfuerzos para atraerlos a sus propias esferas de influencia.

Durante este periodo los defensores de la no-alineación fueron los primeros paises independientes del Sur y Sudeste Asiático, India, Indonesia y Birmania. A principios de 1950 se les unió Yugoslavia, quien formuló una politica exterior similar, basada en la posición especifica que sostuvo en relación a los paises de los grupos de estados orientales y occidentales y de su conflicto mutuo.

La primera declaración oficial de una orientación no-alineada de politica exterior, expresada en India en septiembre de 1946 (por Jawaharlal Nehru, el entonces Primer Ministro del Gobierno Provisional de la India ${ }^{7}$ ), en un momento en que ese pais aún no tenia un status formal independiente, fue pronto seguida por declaraciones sustancialmente similares, formuladas por lideres o por otros gobiernos del Sudeste Asiático. Estos paises, después de comprometerse en la Segunda Guerra Mundial y en los cambios que ella trajo a las relaciones internacionales en todo el mundo, no estaban dispuestos a continuar aceptando el status de paises

${ }^{7}$ Kecsing's Contemporary Archives, $1946-1948$, p. 8169 . 
Bojana Tadic / No-alineación, un vistazo histórico y conceptual

coloniales o semicoloniales del que se habian recién sacudido $y$ proyectiaban la visión de su propio futuro en una dirección diferente. La lucha contra la ocupación japonesa, llevada a cabo en los territorios de estos paises, como una fase de la lucha contra el fascismo, movilizó los movimientos existentes $y$ dio origen a una nueva acción popular que en un principio tuvo carácter emancipador y luego de movimiento anticolonial. La resistencia a los agresores se transformó en una lucha por la total independencia nacional, cuyo objetivo fue comprendido en los primeros años de postguerra, en la mayor parte de esta región. Asi los paises recién independizados del Sur y Sudeste de Asia fueron obligados, en las condiciones de polarización de estados y de intensificación de la Guerra Fria como su fenómeno resultante, a construir su propia posición hacia el mundo en general y a formular una linea básica de sus politicas exteriores.

En esta época la no-alineación tomó la forma de un esfuerzo por preservar la independencia nacional adquirida, y sus componentes, como eran definidos en este periodo, fueron dados a conocer en la siguiente forma: oposición al colonialismo y al racismo, ofrecimiento de apoyo conjunto a los pueblos de Asia y Africa que aún se encontraban bajo el dominio del colonialismo o expuestos a una discriminación de tipo racial; no adhesión al sistema de bloques ni al compromiso en conflictos de estos bloques, los que se habian vuelto más intensos $\gamma$, en consecuencia, of recimiento de una actitud negativa hacia los paises del Occidente, como defensores de la dominación colonialista, $y$ hacia los paises socialistas de Europa Oriental que mostraban reservas hacia las tendencias de desarrollo independiente interno exhibidas por estos paises, y hacia su orientación de la politica exterior. La renuencia para someterse a su linea no significaba, sin embargo, una actitud hostil hacia las grandes potencias. Por el contrario, en las primeras $y$ aun en las más recientes declaraciones hechas por los lideres de estos paises, hubo siempre un permanente deseo de establecer relaciones de amistad y cooperación con todos los paises, incluyendo las anteriores metrópolis y los grandes poderes a condición de que ellas no estuviesen basadas en discriminación de ninguna especie. Finalmente, la no-alineación en ese entonces ya apoyaba a las Naciones Unịdas y se declaraba en favor de la necesidad de organizar la ayuda internacional para el desarrollo económico de los paises subdesarrollados.

El hecho de que en este periodo el proceso de la descolonización obtuviera sus primeros resultados en Asia Sudoriental y que vastas áreas del 


\section{ESTUDIOS I $N$ T ER $\cong$ A C I O $\cong$ A L E S}

continente africano y partes de Asia aún tuvieran un status colonial, hicieron del anticolonialismo el más apremiante problema de los paises fuera de los continentes europeo y americano, la primera plataforma de su asociación $y$ de su actividad internacional conjunta. A este problema se dio tratamiento de prioridad en las primeras reuniones de representantes de estos paises, tales como la Conferencia de las Relaciones Asiáticas celebrada en Nueva Delhi, en 1947, una Segunda Conferencia de los Estados Asiáticos celebrada en la misma ciudad en 1949 y la Conferencia de Colombo en 1954. Esta orientación anticolonialista de los primeros paises independientes de esta área exhibió al mismo tiempo una orientación no comprometida, ya que ofrecia probabilidades de obtener resultados solamente si sus defensores no formaban parte del molde de bloques existentes. Económicamente subdesarrollados, con sistemas politicos inestables, estos paises, al unirse a cualquier bloque podian solamente llegar a ser disciplinados $y$, en algunos casos, subordinados seguidores de las potencias de bloques. La orientación no-bloque, por consiguiente, estaba directamente relacionada con los intereses nacionales inmediatos de estos paises, en los cuales el fortalecimiento de la independencia y la resistencia al colonialismo tenian una prioridad absoluta.

En este periodo hubo también insinuaciones, aunque todavia más bien timidas, de que estos paises aceptarian un papel de mediadores en los conflictos de las grandes potencias, en situaciones relacionadas con los problemas que afectaran sus intereses nacionales. El conflicto de Corea, que trasladó la atmósfera de Guerra Fria al continente asiático, movilizó las actividades diplomáticas y politicas de los paises no-alineados en sistemas de bloques, con miras a conciliar la disputa $y$ a encontrar los medios de terminarla. Gracias a su posición neutral en este conflicto armado y a su independencia respecto a las grandes potencias, estos paises pudieron colaborar en el cese de las operaciones'militarcs. Su contribución fue reconocida cuando se decidió que el representante de la India, pais que tomó parte activa en el proceso de poner término a la guerra de Corea, fuera el presidente de la Comisión de Repatriación de las Naciones Neutrales.

El segundo periodo, en el desarrollo de la no-alineación, de acuerdo a esta clasificación, podria abarcar el lapso de tiempo entre la Conferencia de Bandung, de los paises afroasiáticos, en 1955, y la Conferencia de Belgrado de los Jefes de Estado o Gobiernos de paises no-alineados, efectua- 
da en 1961 . En este periodo el área de la no-alineación creció en gran parte debido a la liberación de los paises africanos, los que en la mayoria de los casos adoptaron la no-alineación como su propia orientación en politica exterior. La acción colectiva de los paises no-alineados llegó a ser una forma más frecuente en su comportamiento y cada vez más presente dentro y fuera de las Naciones Unidas. Su interés empezó a trascender las fronteras nacionales y a trasladarse a un plano internacional más amplio, aunque en la mayoria de los casos fue restringido a un modesto papel de mediador entre las grandes potencias, en su deseo de alentar un acercamiento de sus puntos de vista sobre diferentes problemas internacionales y a iniciativas de carácter de politica proclamada que preparó el camino para la próxima etapa en el desarrollo de la no-alineación. El aumento del número de paises no-alineados y sus iniciativas más frecuentes en el plano internacional, en este periodo, obligó a las grandes potencias a formular su posición frente a esta linea politica. Ya no podian ignorar el hecho de que habia un mundio fuera del suyo que deseaba mantenerse al margen de sus disputas y que no mostraba buena disposición para adoptar sin criticar sus ideologias o para seguir la politica de cualquiera de las grandes potencias o de sus adictos.

Puesto que en el periodo previo la no-alineación tenia ciertos rasgos asiáticos, ya que sus paladines fueron los paises de Asia, en este periodo, caracterizado por el proceso de descolonización, que mientras tanto habia empezado en el continente africano, adquiere ciertos rasgos africanos. Existe sustancialmente la misma tendencia a preservar la independencia nacional, a evitar el comprometerse con los grupos politicos y -a través de una activa participación en los procesos internacionales- a asegurar una posición más favorable para los paises africanos en las relaciones internacionales. Lo que le dio un matiz especial a la no-alineación africana, "o neutralidad positiva" como es frecuentemente descrita en la mayoria de los paises africanos, es que aquello ocurrió y se desarrolló junto con el proceso de formación de la conciencia nacional de las comunidades nacionales, junto con el Movimiento para la Unidad Africana. Dividida por las fronteras administrativas de una colonización europea que no tomó en cuenta las entidades económico-étnicas, ahora, durante el periodo de la descolonización, Africa hubo de enfrentar el proceso de un despertar de la conciencia nacional y de la formación de unidades nacionales. Este proceso se desenvolvió en una base social y cultural me- 
nos desarrollada que en el caso del Asia de los años anteriores, un continente de civilizaciones antiguas y comunidades étnicas más homogéneas.

El movimiento para la unidad de Africa también se desarrolló en paralelo con la descolonización de los paises africanos y dio una huella especial al fenómeno de la no-alineación en esta área. Para muchos de sus movimientos nacionales de liberación, la independencia significaba no sólo la creación de un estado nacional, en el moderno sentido de la palabra, sino también una etapa en el proceso de formar algún tipo de federación africana sobre una base regional o subregional. El Movimiento Panafricano obtuvo su total fuerza durante el decenio de 1950, cuando adquirió el carácter de movimiento nacional masivo. Sin embargo, a pesar de que tuvo una base más amplia, y tradiciones más largas que el Movimiento para la Unidad de los Paises Asiáticos, el proceso de la liberación de Africa, como un resultado de politicas coloniales, cré un gran número de paises pequeños. Africa se fragmentó en un mosaico abigarrado de nuevos estados independientes que, en la mayoria de los casos, seguian teniendo más estrechos contactos con sus antiguas metrópolis que con los paises vecinos, y consecuentemente las fuerzas centrifugas eran muy poderosas. La no-alineación en estas condiciones se identificó con el movimiento para la unidad de paises africanos independientes.

Los acontecimientos importantes desde el punto de vista del desarrollo de la no-alineación en este periodo fueron, primero que todo, la Conferencia de Bandung de los Paises Afroasiáticos, celebrada en abril de 1955, con la participación de representantes de 29 paises de dos continentes; la reunión tripartita de los presidentes Tito y Nasser y el premier Nehru celebrada en el verano de i $95^{6}$ en Brioni, y la xv sesión de la Asamblea General de las Naciones Unidas, en 1960, en la cual 17 nuevos paises de Africa y del Mediterráneo fueron admitidos como miembros de la organización mundial, y a la que concurrió un gran número de estadistas de paises no-alineados de Asia y Africa.

Fue éste el periodo de la primera reunión amplia de los representantes de dos continentes, sobre la base de la cooperación internacional activa cuyos principios, los principios de coexistencia pacifica, fueron adoptados en los documentos finales de esa reunión. A pesar de que en la forma no fue una conferencia de naciones no-alineadas, ya que también concurrieron a ella paises ligados a los bloques de poder por tratados bilaterales o multilaterales, por la tendencia de sus conclusiones, la Conferencia de 
Bojana Tadic / No-alineación, un vistazo histórico y conceptual

Bandung reflejó el espiritu de la no-alineación. Creó condiciones que reforzaron las tendencias antibloques y el progreso de cooperación, prescindiendo de las diferencias ideológicas. Las politicas de coexistencia y anticolonialismo fueron la plataforma que podia reunir las tendencias heterogéneas presentadas en la Conferencia, y la orientación no-alineada, aunque de manera indirecta, estuvo presente en las conclusiones de esta reunión.

Fue también el periodo de los primeros programas de acción de países noalineados, como el caso de la Declaración Brioni, de los Jefes de Estado y Gobierno de Yugoslavia, Egipto e India, en julio de 1956 . Se formó un núcleo de paises no-alineados, los cuales desde entonces y en cooperación con algunos otros, serian los promotores de la mayoria de las acciones internacionales de los paises no-alineados.

Fue éste también el periodo del abandono de iniciativas individuales por los paises no-alineados en las Naciones Unidas, y el de las negativas individuales a ser incorporadas a la Guerra Fria. La no-alineación en la organización del mundo estaba empezando a figurar como un principio que tocaba a un número en aumento de paises que también tomaban parte activa en el proceso de toma de decisiones. Su papel como mediadores en las disputas entre las grandes potencias fue considerado en la decimoquinta sesión de la Asamblea General de las Naciones Unidas, cuando ellos exhortaron a la Unión Soviética y a los Estados Unidos a rènovar contactos en medio de sus situaciones conflictivas en las relaciones interbloques. La iniciativa no tuvo resultado, pero ésta y otras acciones de los paises no-alineados en la organización del mundo, particularmente realizadas en el área de la descolonización afirmaron su presencia y transformaron el patrón politico de las Naciones Unidas, que hasta ese momento habia tendido al bipolarismo.

El tercer periodo, en el desarrollo de la no-alineación abarca el tiempo entre la Conferencia de Belgrado, en t961, y la Conferencia del Cairo de Paises No-alineados, en 1964. Esto puede ser considerado como el climax en la maduración de este fenómeno, caracterizado sobre todo por un relativo alto grado de concordia entre sus partidarios. En este periodo hubo asambleas de naciones no-alineadas a nivel internacional y regional, frecuentes reuniones bilaterales de los estadistas de estos paises, y tentativas para coordinar sus actividades dentro y fuera de las Naciones Unidas. Lo más notable del comportamiento de los paises no-alineados en este 
ESTUDIOS I $\therefore$ TER IA C I O NALES

periodo fue que ya no se limitaron al papel de mediadores para acercar las politicas dispares de los dos grupos dirigentes, aunque este papel continuó como una parte de su actividad, sino que avanzaron hacia la creación de actitudes más independientes en los problemas de relaciones internacionales, e hicieron tentativas de seguir politicas independientes prescindiendo de si coincidian o no, en un momento dado, con las politicas de algunas de las grandes potencias. Finalmente, caracteristica de este periodo fue la expansión adicional del área de no-alineación, porque casi como una regla, todos los paises que adquirieron independencia optaron por esta orientación en politica exterior.

Sin duda que los acontecimientos más significativos desde el punto de vista del desarrollo de la no-alineación en este periodo fueron la Conferencia de Belgrado de Jefes de Estado o Gobiernos de los Paises No-alinealos, en I 961 , la Conferencia Económica del Cairo de los Paiseș Desarrollados, en 1962, y la Conferencia del Cairo de Jefes de Estado y Gobiernos de los Paises No-alineados, en 1964 .

La Primera Reunión Internacional de los Paises No-alineados, ocurrida en Belgrado en septiembre de 1961 , formuló un programa de noalineación y constituyó la hasta entonces más amplia apertura hacia el mundo de los paises no-alineados, lo que puede ser observado al analizar los documentos adoptados por la Conferencia. En la "Declaración sobre el peligro de la guerra y un llamado para la paz", y en idénticos mensajes enviados a los estadistas de la Unión Soviética y de los Estados Unidos dominaba el deseo de los paises no-alineados de enfatizar la amenaza de un conflicto mundial y de animar a las grandes potencias a esforzarse con el fin de alejar esta amenaza. Continuó en estos documentos el papel mediador de los paises no-alineados y su subrayó como uno de sus objetivos el ejercer presión sobre las grandes potencias indicando la posibilidad de su acercamiento y la necesidad de tratar las disputas internacionales por el método de las negociaciones.

El documento más importante de la Conferencia, que en cierto sentido constituye el manifiesto de la no-alineación, formulado por primera vez en un documento internacional, es la Declaración de Jefes de Estado y Gobiernos de Paises No-alineados. Analiza la época contemporánea de las relaciones internacionales, la que se describe como un periodo transitorio desde el antiguo orden, basado en la dominación y el poder, hacia un nuevo orden fundado sobre la cooperación entre las naciones, la 
Bojana Tadić / No-alinención, un vistazo histórico y conceptual

libertad, la igualdad y la justicia. Este periodo se caracteriza por la existencia de bloques militares que evolucionan en grupos militares económicos y politicos más poderosos y que, por la lógica de sus relaciones mutuas, necesariamente causan periódicos empeoramientos en las relaciones internacionales, guerra fria y permanente peligro de transformarla en un conflicto armado. El mundo contemporáneo también se caracteriza por la existencia de diferentes sistemas sociales, los que, de acuerdo a la determinación de la Conferencia, no constituyen un obstáculo infranqueable para la estabilización de la paz, con la condición de que los estados se abstengan de su pretensión de imponer las formas de su propio orden social y su propia politica sobre otros estados, y establezcan relaciones basadas sobre los principios de coexistencia pacifi$\mathrm{ca}$, los que deberian llegar a ser el fundamento de las relaciones internacionales en conjunto. En una situación tal, la extensión del mundo no-alineado fue considerada como la única alternativa posible a la politica de la total división del mundo en bloques, y se miró a los principios de coexistencia pacifica como la única forma posible de relaciones entre estados capaces de asegurar la paz sobre una base duradera. La visión de las relaciones internacionales futuras observada en la Conferencia de Belgrado es la de una comunidad internacional sin relaciones coloniales, con las Naciones Unidas, en cuyos organismos estaria adecuadamente representada la estructura de la comunidad internacional. Un mundo que haria uso de los logros científicos y técnicos de la época con propósitos exclusivamente pacificos y que terminara con la carrera armamentista asegurando el proceso de desarme, un mundo cuyos esfuerzos comunes permitirian el desarrollo de los paises insuficientemente desarrollados. En una palabra, los participantes de la Conferencia de Belgrado proyectaron un nuevo tipo de relaciones internacionales y abogaron por esfuerzos para implementarlas, algunas de las cuales ellos mismos estaban dispuestos a apoyar. En este contexto la no-alineación fue descrita como una politica activa al trascender las relaciones de la Guerra Fria y al promover la cooperación internacional sobre los principios de la coexistencia pacifica ${ }^{8}$.

En contraste con la Conferencia de Belgrado de 1961 , que estableció los

8 Declaración de los Jefes de Estado o Gobiernos de los Paises .'óalineados, La Conferencia de Jefes de Estado o Gobiernos de Paises .lo-alineados, publicado por Publicističko izdavački zavod "Jugoslavija”, Beograd, 1961, pp. 253-261. 
principios básicos de la no-alineación, la Conferencia del Cairo en octubre de 1964 , aceptando del todo estos principios y con una mirada en el desarrollo ulterior de la situación internacional y en su propia determinación de los fenómenos contemporáneos en el mundo, esbozó un programa de acción concreto y comprensivo para los paises no-alineados. El documento final de esta Conferencia, "El Programa para la Paz y la Cooperación Internacional", permanecerá registrado en la historia de la no-alineación como el programa de acción de los paises que adoptaron esta linea politica con miras a la preservación de la paz y el mejoramiento en la posición de los paises en desarrollo, tanto en el plano politico como en el económico.

Finalmente el programa económico de la no-alineación fue elaborado en la Conferencia Económica del Cairo de los Paises en Desarrollo, en julio de 1962 , la que continuó el trabajo empezado en la Conferencia de Belgrado en el terreno de las relaciones internacionales económicas $y$ marcó el comienzo de una actividad organizada de los paises en vias de desarrollo para resolver los problemas de la parte subdesarrollada del mundo, continuada más tarde en la primera y segunda Conferencia de las Naciones Unidas para el Comercio y Desarrollo.

El cuarto y último periodo, en el desarrollo de la no-alineación abarca el periodo más reciente hasta la Conferencia del Cairo de Jefes de Estado o Gobiernos de Paises No-alineados. En esta etapa, más que antes, se destacaron cambios significativos en las relaciones internacionales, marcadas por un cierto relajamiento en las tensiones entre las grandes potencias, fuertes tendencias desintegradoras entre grupos de paises occidentales $y$ orientales, $y$ un simultáneo aumento de presión sobre los nuevos paises liberados. Bajo el efecto de numerosas dificultades internas en los procesos de desarrollo económico y de consolidación politica, y como un resultado de intervenciones externas, muchos paises no-alineados sufrieron durante este periodo crisis internas, inestabilidad politica y levantamientos. Los cambios de regimenes y los golpes militares aumentaron $y$ se hicieron más frecuentes los conflictos entre los mismos paises noalineados o entre estos últimos y otros paises. Todo esto enfatizó la significación de los problemas internos y las relaciones internacionales a nivel regional, en las que los intereses discrepantes de estos paises llegaron a un primer plano. En una situación tal, la no-alineación sufrió un estancamiento $y$ hasta un receso en su dinamismo $y$ en las posibilidades 
Bojana Tadic / No-alincación, un vistazo histórico y conceptual

de la acción común, lo que resultó en la aparición de muchos dilemas de naturaleza teórica y práctica. Estos dilemas, sin embargo, no están relacionados con el problema de si la no-alineación puede sobrevivir en tales condiciones, ya que como una declarada orientación politica nacional exterior, ella rara vez fue puesta en tela de juicio, aun en los periodos de crisis. El problema esencial que aún está presente concierne a la forma y posibilidad de reunir nuevamente a los paises no-alineados en una situación donde hay opiniones divergentes sobre problemas internacionales y tendencias hacia un nuevo aislamiento, presentes en no pocos de estos paises. Estas tendencias aislacionistas fueron causadas por las dificultades de su propia situación, y por una disminución de la confianza en la comunidad internacional y en su prontitud para hacer un esfuerzo y prestar la ayuda necesaria.

\section{III}

- Teniendo en mente la substancia de la no-alineación y los rasgos básicos que reveló en las diferentes fases de su desarrollo y que bosquejamos en esquemas muy generales, volvamos a la pregunta que mencionamos al principio de este estudio: ¿Qué es la no-alineación? o más bien, ¿en qué formas se da a conocer? ¿es esencialmente un acercamiento para la formación de una política exterior, una doçtrina o una filosofia de relaciones internacionales? ¿puede ser considerada como una politica exterior o posee características diferentes?

Es obvio que cualquiera de los tres tipos de respuesta que hemos mencionado, y que se pueden encontrar en numerosas variantes de la literatura teórica sobre este tema, of rece los elementos de una respuesta cuya validez no puede ser discutida, pero dos de ellos vistos separadamente, dan solamente una respuesta parcial a la pregunta.

Primero que todo, hay muchas razones para que la no-alineación sea considerada como una filosofia politica, doctrina o punto de vista mundial ideológico, que gradualmente ha evolucionado y cuya génesis ocurrió en reuniones internacionales de los paises no-alineados. Aunque los elementos de esta doctrina no son idénticos en cuanto a algunos de los paises no-alineados, lo que es comprensible, en vista de las diferencias en las estructuras politicas, en los niveles económicos de desarrollo, herencia cultural, rasgos geográficos y demográficos, etc., es obvio que la no- 
E S T U D I O S I $\therefore$ T E R $\therefore$ A C I O $\therefore$ A L E S

alineación representa una nueva visión de los acontecimientos del mundo contemporáneo, distinto del sistema de concepciones que son caracteristicas para las politicas de poder y esferas de intereses.

Sin embargo, la no-alineación no puede considerarse exclusivamente como una filosofia politica o una visión sobre el mundo de las relaciones internacionales, como lo sostienen la mayoria de los autores que están a favor del primero de los tres modos de pensar. Prescindiendo de la eficiencia y de los resultados obtenidos, y de sus altibajos, la no-alineación representa sin duda un tipo de politica nacional exterior. Sus objetivos estan definidos en los documentos adoptados en las reuniones de paises noalineados o en los documentos oficiales de sus gobiernos y básicamente están diseñados para preservar la independencia nacional y asegurar el desarrollo económico en condiciones de paz, mientras tratan de modificar las relaciones internacionales existentes en esferas politicas y económicas y construir un nuevo tipo de relaciones en la comunidad internacional sobre los principios de coexistencia pacifica y la cooperación activa. Si analizamos el desarrollo de la politica de la no-alineación podemos concluir que estos fines, aunque delineados en diferentes variantes, tienen tendencia a permanecer, lo que presupone la ausencia de cambios en los elementos esenciales de que están compuestos. Esto, sin embargo, no es el caso de las concepciones estratégicas de la politica de la no-alineación, las que, dependiendo de los cambios de las circunstancias en las cuales los paises no-alineados tienen que actuar, evolucionan desde una posición nobloque prevaleciente en las condiciones de una intensa polarización de bloque, hacia el énfasis sobre los problemas económicos, la lucha contra las crecientes presiones externas y las intervenciones de los poderes imperialistas, etc. Por consiguiente, hay suficientes razones para concluir que los fines de la politica de la no-alineación representan, en cierto sentido, las constantes de esta politica, mientras que los elementos estratégicos son categorias cambiantes y están directamente relacionadas con las condiciones concretas en diferentes periodos históricos.

Vista como una politica nacional exterior, la no-alineación no es idéntica en los paises que la han adoptado. Aunque descansa sobre las premisas mencionadas aparece en una variedad de formas, las que en situaciones concretas a veces se apartan de estas premisas. El retraso económico y la inestabilidad politica, como la situación internacional incierta, frecuentemente con- 
Bojana Tadić / No-alineación, un vistazo histórico y conceptual

ducen a una dependencia de la ayuda exterior. A veces esto hace dudar de la implementación de los principios adoptados, por lo que en la politica exterior diaria de un pais no-alineado puede ser considerado como el resultado de los fines adpotados y las posibilidades concretas de su realización.

politica, como la situación internacional incierta, frecuentemente conducen a una dependencia de la ayuda exterior. A veces esto hace dudar de la implementación de los principios adoptados, por lo que en la politica exterior diaria de un pais no-alineado puede ser considerado como el resultado de los fines adoptados y las posibilidades concretas de su realización.

Finalmente, la génesis de la no-alineación muestra que ésta podria no ser definida por la afirmación de que representa un sistema de concepciones acerca del mundo contemporáneo, $y$ una forma de politica exterior basada sobre él. Tampoco podria ser considerada como una sencilla suma de politicas exteriores de los paises no-alineados ni un resultado de politicas individuales conducidas por los paises fuera de las potencias de bloque. El profesor Burton tiene razón al subrayar la posibilidad de definirla como un nuevo sistema internacional en proceso de creación $y$ que constituye un fenómeno cualitativamente nuevo respecto al sistema tradicional de poder en las relaciones internacionales. Es necesario mencionar que en la implementación de este nuevo sistema, cuya base fue sentada por los paises no-alineados, no toman parte permanentemente los mismos paises $y$ además se perciben intensidades variables. En este movimiento que no está institucionalizado, cuyos seguidores son cambiantes, algunos ocasionalmente se alejan, otros llegan. Es un movimiento que posee sus pausas y que registra grandes oscilaciones, como ha sido el caso de los procesos para crear cualquier nueva cualidad en las relaciones internacionales. Pareceria que los éxitos o fallas momentáneas, históricamente consideradas, no son necesariamente importantes para la determinación de su éxito final.

Consecuentemente, la respuesta a la pregunta sobre qué es la no-alineación desde el punto de vista de las formas en las cuales emerge, solamente es posible encontrarla en una sintesis de las respuestas ya mencionadas. Su complejidad refleja la complejidad de un proceso doloroso y largo hacia la superación de las formas de relaciones existentes en la comunidad internacional. 